\title{
Maximal Newton polygons via the quantum Bruhat graph
}

\author{
Elizabeth T. Beazley \\ Department of Mathematics \& Statistics, Williams College, 18 Hoxsey Street, Williamstown, MA 01267, USA
}

\begin{abstract}
This paper discusses a surprising relationship between the quantum cohomology of the variety of complete flags and the partially ordered set of Newton polygons associated to an element in the affine Weyl group. One primary key to establishing this connection is the fact that paths in the quantum Bruhat graph, which is a weighted directed graph with vertices indexed by elements in the finite Weyl group, encode saturated chains in the strong Bruhat order on the affine Weyl group. This correspondence is also fundamental in the work of Lam and Shimozono establishing Peterson's isomorphism between the quantum cohomology of the finite flag variety and the homology of the affine Grassmannian. In addition, using some geometry associated to the poset of Newton polygons, one obtains independent proofs for several combinatorial statements about paths in the quantum Bruhat graph and its symmetries, which were originally proved by Postnikov using the tilted Bruhat order. An important geometric application of this work is an inequality which provides a necessary condition for non-emptiness of certain affine Deligne-Lusztig varieties in the affine flag variety.

Résumé. Cet article étudie une relation surprenante entre la cohomologie quantique de la variété de drapeaux complets et l'ensemble partiellement ordonné de polygones de Newton associé a un élément du groupe de Weyl affine. L'élément clé pour établir cette connexion est le fait que les chemins dans le graphe de Bruhat quantique, qui est un graphe orienté pondéré dont les sommets sont indexés par des éléments du groupe de Weyl fini, encodent des chaînes saturées dans l'ordre de Bruhat fort sur le groupe de Weyl affine. Cette correspondence est aussi fondamentale dans les travaux de Lam et Shimonozo qui établissent l'isomorphisme de Peterson entre la cohomologie quantique de la variété de drapeaux finie et l'homologie de la Grassmannienne affine. De plus, en utilisant la géométrie associée a l'ensemble partiellement ordonné des polygones de Newton, on obtient des preuves indépendentes pour plusieurs assertions combinatoires sur les chemins dans le graphe de Bruhat quantiques et les symétries de ce graphe, qui ont été originellement démontrées par Postnikov en utilisant l'ordre de Bruhat incliné. Une application géometrique importante de ce travail est une inégalité qui donne une condition nécessaire pour que certaines variétés de Deligne-Lusztig affines dans la variété de drapeaux affine soient non-vides.
\end{abstract}

Keywords: quantum Bruhat graph, quantum Schubert calculus, affine Weyl group, Newton polygon, affine DeligneLusztig variety, Mazur's inequality

\section{Introduction}

This paper investigates connections between the geometry and combinatorics in two different, but surprisingly related contexts: families of subvarieties of the affine flag variety in characteristic $p>0$ and the quantum cohomology of the complex flag variety. We establish explicit combinatorial connections between fundamental questions in these two theories using paths in the quantum Bruhat graph as a primary 
tool. We begin with a brief historical survey of each of these two geometric contexts and the relevant combinatorial questions, and then we informally state our main result.

In the 1950s, Dieudonné introduced the notion of isocrystals over perfect fields of characteristic $p>0$ (see [Man63]), which Grothendieck extended to families of F-crystals in [Gro74]. Isogeny classes of $F$-crystals are indexed by combinatorial objects called Newton polygons, a family of lattice polygons in the plane. Kottwitz used the theory of algebraic groups to explicitly study the set of Newton polygons associated to any connected reductive group $G$ over a discretely valued field. In particular, he observed that there is a natural bijection between the set of Frobenius-twisted conjugacy classes in $G$ and a suitably generalized notion of the set of Newton polygons; see [Kot85] and [Kot97]. The poset of Newton polygons in the context of reductive group theory has interesting combinatorial and Lie-theoretic interpretations, which were first described by Chai in [Cha00]. For example, Chai proves that the poset of Newton slope sequences is ranked; i.e., any two maximal chains have the same length. In addition to the classification of $F$-crystals and Frobenius-twisted conjugacy classes, modern interest in the poset of Newton polygons is motivated by applications to the study of two important families of varieties in arithmetic algebraic geometry: affine Deligne-Lusztig varieties (see [GHKR10]) and Shimura varieties (see [Rap05]).

Completely independently, physicists working in the field of superstring theory in the early 1990s observed that certain algebraic varieties seemed to present natural vacuum solutions to superstring equations, and thus developed a theory of quantum cohomology; see [Wit95]. Using the notion of mirror symmetry, they were able to employ this cohomological framework to calculate the number of rational curves of a given degree on a general quintic hypersurface in projective 4-space; see [CdlOGP91]. Mathematicians first rigorously worked out the structure of the quantum cohomology ring for the Grassmanian variety of $k$-planes in complex $n$-dimensional space (see [BDW96]), and initial mathematical applications were also to enumerative geometry. Modern mathematical interest focuses on concretely understanding the structure of the quantum cohomology ring for any homogeneous variety $G / P$, where $G$ is a complex reductive algebraic group and $P$ a parabolic subgroup. The ring $Q H^{*}(G / P)$ has a basis of Schubert classes, indexed by elements of the corresponding Weyl group. The driving question in the field is to find non-recursive, positive combinatorial formulas for expressing the quantum product of two Schubert classes in terms of this basis. Applications include statistics about mapping projective curves to $G / P$ satisfying various incidence conditions, but the impact now extends beyond enumerative geometry into many other aspects of algebraic geometry, combinatorics, representation theory, number theory, and physics.

The main result in this paper shows that there is a closed combinatorial formula for the maximal element in the poset of Newton polygons associated to a fixed affine Weyl group element in terms of paths in the quantum Bruhat graph, which is a directed graph with vertices indexed by the elements of the Weyl group and weights given by the reflections used to get from one element to the other. Here we informally state our main result.

Main Theorem. Let $x$ be an element of the affine Weyl group, and assume that the associated alcove is far enough away from the walls of any Weyl chamber. Let $w$ denote the finite part of $x$, and $v$ the Weyl chamber in which $x$ lies. Then the maximal Newton polygon is given by taking the translation part of $x$ and subtracting the coroot corresponding to the weight of any path of minimal length in the quantum Bruhat graph from $v w_{0}$ to $w^{-1} v w_{0}$, where $w_{0}$ is the longest element in the finite Weyl group.

The weight of a path in the quantum Bruhat graph is defined to be the sum of the coroots corresponding to the reflections labeling any downward edges in the path. For a precise statement, see Theorem 4.1 


\section{Notation}

Let $G$ be a split connected reductive group, and $B$ a fixed Borel subgroup with $T$ a maximal torus in $B$. The finite Weyl group $W$ is the quotient $N_{G}(T) / T$. Denote by $\ell: W \rightarrow \mathbb{Z}_{>0}$ the length function, and by $w_{0}$ the element of longest length. Let $R=R^{+} \cup R^{-}$be the set of roots, and $s_{\alpha}$ the reflection in $W$ corresponding to the positive root $\alpha \in R^{+}$. Let $\Delta=\left\{\alpha_{i} \mid i \in I\right\}$ be an ordered basis of simple roots, and $\left\{\alpha_{i}^{\vee} \mid i \in I\right\}$ a basis of simple coroots. Denote by $s_{\alpha_{i}}=s_{i}$ the corresponding simple reflection in $W$. Let $\rho$ denote the half-sum of the positive roots. Denote by $Q=\bigoplus \mathbb{Z} \alpha_{i}$ and $Q^{\vee}=\bigoplus \mathbb{Z} \alpha_{i}^{\vee}$ the root and coroot lattices. Let $\omega_{\alpha}$ be the fundamental weight corresponding to $\alpha$, which is dual to $\alpha^{\vee}$ with respect to the inner product $\langle\cdot, \cdot\rangle \rightarrow \mathbb{Z}$. Let $\Lambda$ denote the weight lattice, which is the $\mathbb{Z}$-span of the fundamental weights. We say that $\lambda \in Q^{\vee}$ is dominant if $\left\langle\lambda, \alpha_{i}\right\rangle \geq 0$ for all $i \in I$. Denote by $Q^{+}$the set of dominant elements of $Q^{\vee}$, and $\Lambda^{+}$the set of dominant weights. We say that $\lambda \in Q^{\vee}$ is regular if the stabilizer of $\lambda$ in $W$ is trivial. The coroot $\lambda$ is superregular if $|\langle\lambda, \alpha\rangle| \geq M$ for all $\alpha \in R^{+}$for some fixed sufficiently large $M$.

The affine Weyl group corresponding to $W$ is the semi-direct product $\widetilde{W}=Q^{\vee} \rtimes W$. For $\lambda \in Q^{\vee}$, let $t^{\lambda}$ be the image of $\lambda$ in $\widetilde{W}$ so that any $x \in \widetilde{W}$ may be written as $x=t^{\lambda} w$ for some $\lambda \in Q^{\vee}$ and $w \in W$. By $\lambda^{+}$we mean the unique dominant coroot in the $W$-orbit of $\lambda \in Q^{\vee}$. Then $t^{v \lambda}=v^{-1} t^{\lambda} v$, and so if $\lambda$ is regular then there exists a unique $v \in W$ such that $t^{\lambda} w=t^{v \lambda^{+}} w$.

\section{The Poset of Newton Polygons}

In the context of Newton polygons, we will work with a split connected reductive group $G$ over the discretely valued field $F=\overline{\mathbb{F}_{q}}((t))$ for $q=p^{k}$, which has characteristic $p>0$. The ring of integers in $F$ then equals the ring of formal power series $\mathcal{O}=\overline{\mathbb{F}_{q}}[[t]]$. We can extend the usual Frobenius automorphism $x \mapsto x^{q}$ on $\overline{\mathbb{F}_{q}}$ to a map $\sigma: F \rightarrow F$ by allowing $\sigma$ to act on the coefficients: $\sum a_{i} t^{i} \mapsto \sum a_{i}^{q} t^{i}$.

\subsection{Newton polygons from isocrystals}

Definition 3.1. An isocrystal $(V, \Phi)$ is a finite-dimensional vector space $V$ over $F$ together with a $\sigma$-linear bijection $\Phi: V \rightarrow V$; that is, $\Phi(a v)=\sigma(a) \Phi(v)$ for $a \in F$ and $v \in V$.

A simple example of an isocrystal is $\left(F^{n}, \Phi\right)$, where $\Phi=A \circ \sigma$ for some $A \in G L_{n}(F)$, and $\sigma$ acts on $V$ coordinate-wise. Similarly, for $G$ any connected reductive group over $F$, any element $g \in G(F)$ determines an isocrystal over $F$; see [Kot85] or [RR96] for the details. Conversely, if we fix a basis $\left\{e_{1}, \ldots, e_{n}\right\}$ for $V$, then note that we can associate a matrix $A \in G L_{n}(F)$ to $(V, \Phi)$ defined by $\Phi\left(e_{i}\right)=$ $\sum_{i=1}^{n} A_{j i} e_{j}$, in which case we write $\Phi=A \circ \sigma$ for $A=\left(A_{i j}\right)$.

Dieudonné showed that the category of isocrystals over $F$ is semi-simple, and that the simple objects are naturally indexed by $\mathbb{Q}$. That is, any isocrystal $(V, \Phi)$ is isomorphic to a direct sum $\oplus_{i=1}^{n} V_{s_{i} / r_{i}}$ where $\operatorname{gcd}\left(s_{i}, r_{i}\right)=1$ and the $V_{s_{i} / r_{i}}$ are simple; for a proof that this decomposition exists, see [Dem72].

Definition 3.2. If $(V, \Phi)$ is an $n$-dimensional isocrystal over $F$ such that $V=\oplus_{i=1}^{n} V_{s_{i}} / r_{i}$, then the Newton slope sequence for $(V, \Phi)$ is $\lambda=\left(\lambda_{1}, \ldots, \lambda_{n}\right) \in Q^{+} \otimes_{\mathbb{Z}} \mathbb{Q}$ where each $s_{i} / r_{i}$ is repeated $r_{i}$ times and the $\lambda_{i}$ are ordered so that $\lambda_{1} \leq \cdots \leq \lambda_{n}$.

Definition 3.3. The Newton polygon for an $n$-dimensional isocrystal $(V, \Phi)$ is the graph of the function 


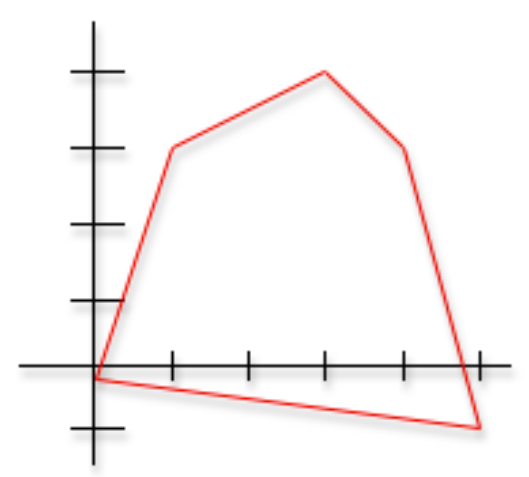

Fig. 1: A Newton polygon in $G L_{5}(F)$.

$\bar{\nu}:[0, n] \longrightarrow \mathbb{R}$ given by

$$
\begin{cases}\bar{\nu}(i)=0, & \text { if } i=0 \\ \bar{\nu}(i)=\lambda_{1}+\cdots \lambda_{i}, & \text { if } i=1, \ldots, n,\end{cases}
$$

and then extended linearly between successive integers. Denote by $\mathcal{N}(G)$ the set of all possible Newton polygons for isocrystals arising from elements $g \in G(F)$, and by $\nu: G \longrightarrow \mathcal{N}(G)$ the Newton map.

For example, the Newton polygon in Figure 1 corresponds to a 5-dimensional isocrystal having Newton slope sequence $\lambda=\left(3, \frac{1}{2}, \frac{1}{2},-1,-4\right)$. Because a Newton slope sequence $\lambda$ uniquely determines a Newton polygon and vice versa, we will occasionally abuse terminology and refer to elements $\lambda \in \mathcal{N}(G)$ interchangeably as both polygons in the plane and slope sequences in $Q^{+} \otimes_{\mathbb{Z}} \mathbb{Q}$.

If $g$ is an element of the affine Weyl group $\widetilde{W}$ viewed as a subgroup of $G(F)$, then there is a shortcut for computing the Newton polygon $\nu(g)$. For our purposes, this is the most important special case, and so we highlight this example as a separate statement.

Proposition 3.4. Let $y=t^{\lambda} w \in \widetilde{W}$, and suppose that the order of $w$ in $W$ equals $k$. Then the Newton slope sequence for y equals

$$
\nu(y)=\left(\frac{1}{k} \sum_{i=1}^{k} w^{i}(\lambda)\right)^{+} .
$$

This formula is a standard fact, the proof of which can be found in [GHKR10], but we provide an explicit example for clarity. Let $G=S L_{3}(F)$ so that $W=S_{3}$ is generated by the two simple reflections $s_{1}$ and $s_{2}$. Let $y=t^{(-2,0,2)} s_{1} \in \widetilde{S_{3}}$. Then the Newton slope sequence for $y$ equals $\nu(y)=$ $\left[\frac{1}{2}((0,-2,2)+(-2,0,2))\right]^{+}=\left[\frac{1}{2}(-2,-2,4)\right]^{+}=(2,-1,-1)$, and the corresponding Newton polygon is the convex hull of the three points $(0,0),(1,2)$, and $(3,0)$.

\subsection{Newton polygons from characteristic polynomials}

We now provide a more concrete equivalent definition of the Newton polygon associated to an element $g \in G(F)$, although we remark that Definition 3.3 is the one found in the overwhelming majority of the literature. 
Define a non-commutative ring $R=F[\sigma]$, where for $a \in F$ we have that $\sigma a=\sigma(a) \sigma$. Given an isocrystal $(V, \Phi)$ over $F$, identifying $\sigma^{i} v:=\Phi^{i}(v)$ makes $V$ into an $R$-module. It turns out that in fact $(V, \Phi)$ is a cyclic module over the ring $R$; that is, $R v=V$ for some $v$ in $V$. In this context, we call the generator $v$ a cyclic vector. Upon choosing a cyclic vector $v$, we may thus write $V \cong R / R f$ for some $f=\Phi(v)^{n}+a_{1} \Phi(v)^{n-1} \cdots+a_{n-1} \Phi(v)+a_{n} v$ for some $a_{i} \in F$, where $n=\operatorname{dim}_{F}(V)$. We shall call $f$ the characteristic polynomial associated to $(V, \Phi, v)$.

Definition 3.5. The Newton polygon of $(V, \Phi, v)$ is the tightest-fitting convex polygon joining the points $(0,0)$ and $\left(n, \operatorname{val}\left(a_{n}\right)\right)$ that passes either through or above all of the points in the set $\left\{(0,0),\left(i, \operatorname{val}\left(a_{i}\right)\right)\right\}$ for $i=1, \ldots, n$. Here, the $a_{i}$ are the coefficients of the characteristic polynomial $f$, and $\operatorname{val}\left(a_{i}\right)$ is the valuation map which picks out the smallest power of $t$ occurring in $a_{i} \in F$; define val $(0)=-\infty$.

Although $f$ depends on the choice of a cyclic vector, the Newton polygon associated to $(V, \Phi, v)$ is independent of the choice of cyclic vector $v$.

Example 3.6. Let $\left(F^{2}, \Phi\right)$ be an isocrystal, where $\Phi=g \circ \sigma$ for $g=\left(\begin{array}{cc}t^{2} & t \\ 1 & t^{3}\end{array}\right)$. Then one can show that $e_{1}$ is a cyclic vector for $\left(F^{2}, \Phi\right)$ by observing that $\Phi\left(e_{1}\right) \wedge e_{1} \neq 0$. We can then compute that the characteristic polynomial for $\left(F^{2}, \Phi, e_{1}\right)$ equals

$$
\Phi^{2}\left(e_{1}\right)+\left(-t^{2}-t^{3}\right) \cdot \Phi\left(e_{1}\right)+\left(-t+t^{5}\right) \cdot e_{1}=0 .
$$

Therefore, $\operatorname{val}\left(a_{1}\right)=2$ and $\operatorname{val}\left(a_{2}\right)=1$ so that the Newton polygon $\nu(g)$ is the convex hull of the three points $(0,0),(1,2)$, and $(2,1)$ and has slope sequence $(2,-1)$.

Remark 3.7. We point out that using either definition of the Newton polygon, there are challenges in explicitly computing $\nu(g)$ given a generic element $g \in G(F)$. Definition 3.5 is quite concrete, although calculations such as those in Example 3.6 become unwieldy for groups of large rank; see [Bea09] for the case of $G=S L_{3}$. On the other hand, using Definition 3.3 requires a detailed understanding of the category of isocrystals and its simple objects.

\subsection{The partial ordering on $\mathcal{N}(G)$}

There is a natural partial ordering on the set of Newton polygons $\mathcal{N}(G)$. Given a Newton polygon $\lambda$ in the plane, we say that another Newton polygon $\lambda^{\prime}$ satisfies $\lambda^{\prime} \leq \lambda$ if they share a left and rightmost vertex and all other edges of $\lambda^{\prime}$ lie either on or below those of $\lambda$. Equivalently, we may compare two Newton slope sequences $\lambda, \lambda^{\prime} \in Q^{+} \otimes_{\mathbb{Z}} \mathbb{Q}$ by using the dominance order; i.e., $\lambda^{\prime} \leq \lambda$ if $\lambda-\lambda^{\prime}$ is a non-negative linear combination of positive coroots. For example, $\mu=(2,1,0,0,-4) \leq\left(3, \frac{1}{2}, \frac{1}{2},-1,-4\right)$ since all partial sums for the slope sequence of the Newton polygon in Figure 1 are greater than or equal to those for $\mu$.

The full poset of Newton polygons $\mathcal{N}(G)=\{\nu(g) \mid g \in G\}$ was studied by Kottwitz in [Kot85] and [Kot97], Rapoport-Richartz in [RR96], and Chai in [Cha00] from the perspective of arithmetic algebraic geometry. For example, Chai proves that the poset $\mathcal{N}(G)$ is ranked; i.e. all maximal chains have the same length, and that $\mathcal{N}(G)$ is a lattice. Together with Luis Serrano, the author is working to further develop the combinatorics of the poset of Newton polygons, beginning by understanding the topology of the associated order complex; see [BS12]. 


\subsection{Newton polygons associated to an affine Weyl group element}

Motivated by applications to Shimura varieties and affine Deligne-Lusztig varieties, it is also important to study the combinatorics of subsets of $\mathcal{N}(G)$ in which one restricts to Newton polygons associated to a fixed element of the affine Weyl group $\widetilde{W}$.

One analog of the Bruhat decomposition on $G(\mathbb{C})$ in the affine context says that $G(F)=\coprod_{x \in \widetilde{W}} I x I$, where $I$ is the Iwahori subgroup. The Iwahori subgroup $I$ of $G(F)$ is defined to be the inverse image of $B$ under the map $G\left(\overline{\mathbb{F}_{q}}[[t]]\right) \rightarrow G\left(\overline{\mathbb{F}_{q}}\right)$. For example, if $G=S L_{n}(F)$, then

$$
I=\left(\begin{array}{cccc}
\mathcal{O}^{\times} & \mathcal{O} & \ldots & \mathcal{O} \\
t \mathcal{O} & \mathcal{O}^{\times} & \ldots & \mathcal{O} \\
\vdots & \vdots & \ddots & \vdots \\
t \mathcal{O} & t \mathcal{O} & \ldots & \mathcal{O}^{\times}
\end{array}\right)
$$

The question then becomes to study

$$
\mathcal{N}(G)_{x}=\{\nu(g) \mid g \in I x I\},
$$

the set of Newton polygons arising for elements in the "affine Bruhat cell" IxI. These subsets clearly inherit the partial ordering on $\mathcal{N}(G)$.

The posets $\mathcal{N}(G)_{x}$ have only been fully computed for groups of low rank or when $x$ has a special form, but many nice combinatorial properties of $\mathcal{N}(G)$ also hold for $\mathcal{N}(G)_{x}$ in these cases. For example, in [Bea09] it is proved that if $G=G L_{2}$ or $G=S L_{3}$, the poset $\mathcal{N}(G)_{x}$ is a ranked lattice. As another example, if $x=t^{\lambda}$ is a pure translation element, then $\mathcal{N}(G)_{x}=\left\{\lambda^{+}\right\}$; see [GHKR10]. The precise relationship between $\mathcal{N}(G)_{x}$ and $\mathcal{N}(G)$ remains quite opaque. For example, it is not even known for which $x$ the poset $\mathcal{N}(G)_{x}$ is a subinterval of $\mathcal{N}(G)$.

\subsection{Maximal Newton polygons}

The poset $\mathcal{N}(G)_{x}$ is always a finite set, and we now show that it has a unique maximal element. For any fixed $x \in \widetilde{W}$, the double coset $I x I$ is irreducible. If we denote by $G_{\lambda}=\{g \in G \mid \nu(g)=\lambda\}$, then we can consider the intersections $(I x I)_{\lambda}:=G_{\lambda} \cap I x I$. Then $I x I$ is the finite union of subsets of the form $(I x I)_{\lambda}$, any two of which are disjoint. If $\lambda \in \mathcal{N}(G)_{x}$ is maximal, then $(I x I)_{\lambda}$ is actually an open subset of $I x I$. But since $I x I$ is irreducible, there must exist a unique maximal element in $\mathcal{N}(G)_{x}$. Therefore, the following is well-defined.

Definition 3.8. Given $x \in \widetilde{W}$, we define the maximal Newton polygon, or equivalently, the maximal Newton slope sequence $\nu_{x} \in Q^{+} \otimes_{\mathbb{Z}} \mathbb{Q}$, to be the unique maximal element in $\mathcal{N}(G)_{x}$; i.e., $\nu_{x}$ is defined such that for all $\lambda \in \mathcal{N}(G)_{x}$, we have $\lambda \leq \nu_{x}$.

The first combinatorial formula for the unique maximal element $\nu_{x}$ in $\mathcal{N}(G)_{x}$ was discovered by [Vie09] and may be written as follows.

Theorem 3.9 (Viehmann). Given $x \in \widetilde{W}$,

$$
\nu_{x}=\max \{\nu(y) \mid y \in \widetilde{W} \text { s. } t . y \leq x\},
$$

where the maximum is taken with respect to dominance order and the elements $y$ and $x$ are related by strong Bruhat order. 
Although elegant, the disadvantage of the above formula is that it is difficult to actually use without the aid of a computer, involving both computing every element less than the fixed affine Weyl group element $x$ in Bruhat order, and then computing the Newton polygon for each of those elements. Our main result, which is formally stated in Section 4.1, provides a closed combinatorial formula for the maximal Newton polygon associated to an affine Weyl group element which may be easily computed by hand.

\section{Quantum Schubert Calculus}

The main result in this paper shows that there is a closed combinatorial formula for maximal Newton polygons in terms of paths in the quantum Bruhat graph, a labeled directed graph with vertices in the finite Weyl group. The nomenclature comes from the fact that this graph was introduced by Brenti, Fomin, and Postnikov to capture the multiplicative structure of the quantum cohomology ring of the complex flag manifold [BFP99]. Using the quantum Bruhat graph to answer questions about the poset of Newton polygons in turn leads to several results in quantum Schubert calculus, which are stated in Section 4.2

\subsection{The definition of the quantum Bruhat graph}

Here we formally define the quantum Bruhat graph, which will be our primary combinatorial tool. The vertices are given by the elements of the finite Weyl group $w \in W$. Two elements are connected by an edge if they are related by a reflection satisfying one of two "quantum relations." More precisely, there is a directed edge $w \longrightarrow w s_{\alpha}$ if one of two length relationships between $w$ and $w s_{\alpha}$ is satisfied:

$$
\begin{array}{ll}
\ell\left(w s_{\alpha}\right) & =\ell(w)+1, \text { or } \\
\ell\left(w s_{\alpha}\right) & =\ell(w)-\left\langle\alpha^{\vee}, 2 \rho\right\rangle+1 .
\end{array}
$$

The first type of edges are simply those in the usual Hasse diagram for the strong Bruhat order on $W$. The second type of edges, all of which are directed downward in the graph, are "quantum" edges coming from the quantum Chevalley-Monk formula of Peterson [Pet96]. The edges are then labeled by the reflection used to get from one element to the other, so that the edge from $w \longrightarrow w s_{\alpha}$ is labeled by the reflection $s_{\alpha}$. Figure 2 shows the quantum Bruhat graph for $W=S_{3}$.

We now define the weight of any path in the quantum Bruhat graph. For an edge $w \longrightarrow w s_{\alpha}$ resulting from the relation $\ell\left(w s_{\alpha}\right)=\ell(w)+1$, there is no contribution to the weight. On the other hand, an edge arising from the relation $\ell\left(w s_{\alpha}\right)=\ell(w)-\left\langle\alpha^{\vee}, 2 \rho\right\rangle+1$ contributes a weight of $\alpha^{\vee}$. The weight of a path in the quantum Bruhat graph is then defined to be the sum of the weights of the edges in the path. For example, in the quantum Bruhat graph for $W=S_{3}$ depicted in Figure 2 the weight of any path of minimal length from $s_{1} s_{2}=\left[\begin{array}{lll}2 & 3 & 1\end{array}\right]$ to $s_{2}=\left[\begin{array}{lll}1 & 3 & 2\end{array}\right]$, all of which have length 3, would equal $\alpha_{1}^{\vee}+\alpha_{2}^{\vee}$.

We are now prepared to formally state our main result, which provides a readily computable closed combinatorial formula for the maximal Newton polygon $\nu_{x} \in \mathcal{N}(G)_{x}$. The general shape of $\nu_{x}$ for $x=t^{v \lambda^{+}} w$ is that $\nu_{x}=\lambda^{+}-\mu$, where $\mu$ is a correction factor obtained by looking at the weight of any minimal length path in the quantum Bruhat graph between two elements which are uniquely determined by $w$ and $v$.

Theorem 4.1. Let $x=t^{v \lambda^{+}} w \in \widetilde{W}$, and assume that $\lambda$ is superregular. Then

$$
\nu_{x}=\lambda^{+}+w_{0}\left(\alpha_{d}^{\vee}\right)
$$

where $\alpha_{d}^{\vee}$ is the weight of any path of minimal length in the quantum Bruhat graph from vw $w_{0}$ to $w^{-1} v w_{0}$. 


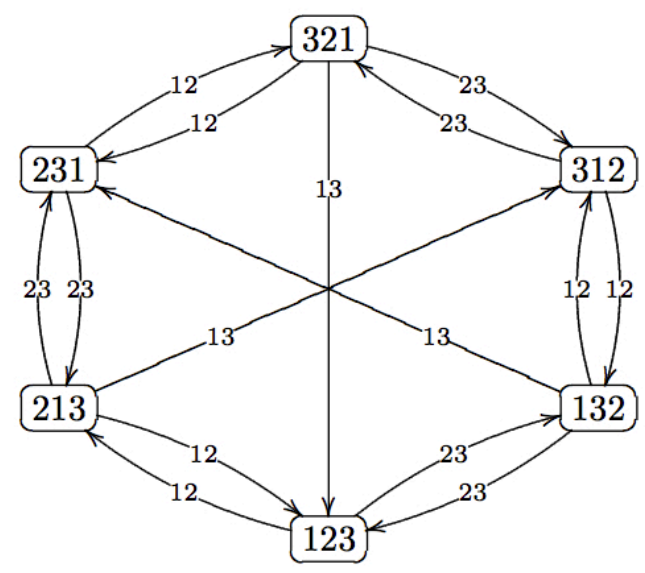

Fig. 2: The quantum Bruhat graph for $S_{3}$.

Because $\alpha_{d}^{\vee}$ will always be a non-negative sum of positive coroots, the quantity $w_{0}\left(\alpha_{d}^{\vee}\right)$ will actually be a non-positive sum of positive coroots so that $\nu_{x} \leq \lambda^{+}$. We point out in addition that Conjecture 2 from [Bea09] follows as a direct corollary.

\subsection{Results in quantum Schubert calculus}

We now discuss the surprising connections between this result about Newton polygons, arising from geometric questions about affine flag varieties in characteristic $p>0$, to the quantum cohomology of standard complete flag varieties over $\mathbb{C}$. Given a complex reductive group $G$, the classical cohomology of the complete flag variety $G / B$ over $\mathbb{C}$ is a free $\mathbb{Z}$-module generated by Schubert classes, which are indexed by elements in the Weyl group $W$. If we define $\mathbb{Z}[q]:=\mathbb{Z}\left[q_{1}, \ldots, q_{r}\right]$, where $r$ is the rank of $G$, the quantum cohomology ring of $G / B$ then equals $Q H^{*}(G / B)=H^{*}(G / B, \mathbb{Z}) \otimes_{\mathbb{Z}} \mathbb{Z}[q]$ as a $\mathbb{Z}[q]$ module, and will also have a $\mathbb{Z}[q]$-basis of Schubert classes $\sigma_{w}$ where $w \in W$. The main problem in modern quantum Schubert calculus is to explicitly compute the products

$$
\sigma_{u} * \sigma_{v}=\sum_{w, d} c_{u, v}^{w, d} q^{d} \sigma_{w}
$$

by finding non-recursive, positive combinatorial formulas for the Gromov-Witten invariants $c_{u, v}^{w, d}$ and the quantum parameters $q^{d}$.

Postnikov strengthens a result of Fulton and Woodward providing a combinatorial formula for the unique minimal monomial $q^{d}$ which occurs with nonzero coefficient in any quantum Schubert product; see [Pos05] and [FW04]. The following theorem relates these results in quantum Schubert calculus to finding maximal Newton polygons.

Theorem 4.2. Assume that $\lambda$ is superregular. Then $q^{d}$ is the minimal monomial in the quantum product $\sigma_{u} * \sigma_{v}$ if and only if $\lambda^{+}+w_{0}\left(\alpha_{d}^{\vee}\right)$ is the maximal Newton polygon in $\mathcal{N}(G)_{x}$, where $x=t^{u w_{0}\left(\lambda^{+}\right)} u v^{-1} w_{0}$. 
The method for proving Theorem 4.1 also provides independent proofs of several combinatorial statements about paths in the quantum Bruhat graph which originally appeared in [Pos05].

Theorem 4.3. Let $u, v \in W$ be any Weyl group elements. Then:

1. There exists a directed path from $u$ to $v$ in the quantum Bruhat graph.

2. All shortest paths from $u$ to $v$ have the same weight, say $d_{\text {min }}$.

3. If $d$ is the weight of any path from $u$ to $v$, then $q^{d}$ is divisible by $q^{d_{\text {min }}}$.

Here, writing $\alpha_{d}^{\vee}=d_{1} \alpha_{1}^{\vee}+\cdots+d_{r} \alpha_{r}^{\vee}$ as a sum of coroots and defining $d=\left(d_{1}, \ldots, d_{r}\right) \in \mathbb{Z}^{r}$, then by $q^{d}$ we mean $q_{1}^{d_{1}} \cdots q_{r}^{d_{r}}$. The key idea is to realize saturated chains in affine Bruhat order in terms of paths in the quantum Bruhat graph, as discussed in Section 6, whereas Postnikov's original proof relies on the combinatorics of the tilted Bruhat order from [BFP99].

In addition, using the automorphisms of the Iwahori subgroup discussed in [Bea09], it is also possible to provide independent geometric proofs of the symmetries of the quantum Bruhat graph for $W=S_{n}$ appearing in [Pos01], which in turn correspond to symmetries of the Gromov-Witten invariants themselves. These additional results may be found in the full version of this paper.

\section{Affine Deligne-Lusztig Varieties and Mazur's Inequality}

We now discuss the relationship between Theorem 4.1 and an important family of subvarieties in the affine flag variety in positive characteristic. In [DL76] and [Lus78], Deligne and Lusztig constructed a family of varieties $X_{w}$ for $w \in W$ to study the representation theory of finite Chevalley groups. Affine Deligne-Lusztig varieties, which are generalizations of these classical Deligne-Lusztig varieties, have geometric properties which are governed by subtle combinatorics of the affine Weyl group. For $x \in \widetilde{W}$ and $b \in G(F)$ where again $F=\overline{\mathbb{F}_{q}}((t))$, the associated affine Deligne-Lusztig variety is defined as

$$
X_{x}(b):=\left\{g \in G(F) / I \mid g^{-1} b \sigma(g) \in I x I\right\} .
$$

It is useful to study affine Deligne-Lusztig varieties because they are intimately related to reductions modulo $p$ of Shimura varieties, which lie at the heart of the Langlands program; see [Rap05].

Unlike in the classical case in which Lang's Theorem automatically says that $X_{w}$ is non-empty for every $w \in W$, affine Deligne-Lusztig varieties frequently tend to be empty. Providing a complete characterization for the pairs $(x, b)$ for which the associated affine Deligne-Lusztig variety is non-empty as a set has proven to be a delicate combinatorial problem. In the context of affine Deligne-Lusztig varieties inside the affine Grassmannian, the non-emptiness question is phrased in terms of Mazur's inequality, which relates the coroot $\lambda$ from the torus part of the affine Weyl group element and the Newton polygon of $b$; see [Maz72] and [Kat79]. If $\nu(b)$ denotes the Newton polygon associated to $b$, Mazur's inequality roughly states that $\nu(b) \leq \lambda^{+}$in dominance order; i.e. the difference $\lambda^{+}-\nu(b)$ is a non-negative linear combination of positive coroots.

Although no simple analog of Mazur's inequality perfectly predicts whether or not $X_{x}(b)$ is non-empty, Theorem 4.1 can be thought of as an Iwahori analog of Mazur's inequality, which provides a necessary condition for non-emptiness under a superregularity hypothesis on the coroot. 
Theorem 5.1. Let $x=t^{v \lambda^{+}} w \in \widetilde{W}$, and assume that $\lambda$ is superregular. Fix $b \in G(F)$, and let $\nu(b)$ denote the Newton polygon for $b$. If $X_{x}(b)$ is non-empty, then

$$
\nu(b) \leq \lambda^{+}+w_{0}\left(\alpha_{d}^{\vee}\right)
$$

where $d$ is the weight of any path of minimal length in the quantum Bruhat graph from $v w_{0}$ to $w^{-1} v w_{0}$.

Because $\alpha_{d}^{\vee}$ will always be a non-negative sum of positive coroots, the quantity $w_{0}\left(\alpha_{d}^{\vee}\right)$ will be a nonpositive sum of positive coroots so that actually $\nu(b) \leq \lambda^{+}+w_{0}\left(\alpha_{d}^{\vee}\right) \leq \lambda^{+}$. As such, this theorem is a partial generalization of Mazur's inequality on the affine Grassmannian to the case of the full affine flag variety.

\section{The quantum Bruhat graph and affine Bruhat order}

In this section, we outline the main ingredients which enter into the proof of Theorem 4.1 First, recall that given an element $y=t^{\lambda} w$ in the affine Weyl group, Mazur's inequality says that $\nu(y) \leq \lambda^{+}=\nu\left(t^{\lambda}\right)$. Generically, however, the Newton polygon of an affine Weyl group element whose finite part is non-trivial will lie strictly below the Newton polygon of the corresponding pure translation element.

Proposition 6.1. If $y=t^{\lambda} w \in \widetilde{W}$ is such that $\lambda$ is regular and $w$ is non-trivial, then $\nu(y)<\nu\left(t^{\lambda}\right)$.

Now recall that Equation (3.6) says that to compute $\nu_{x}$ we should take a maximum in dominance order over all $\nu(y)$ such that $y \leq x$. The point of Proposition 6.1 is that we can reduce our problem to finding the largest pure translation element in Bruhat order which is less than or equal to $x$.

Proposition 6.2. There exists an $M \in \mathbb{Z}_{\geq 0}$ so that given any $\lambda \in Q^{\vee}$ such that $|\langle\lambda, \alpha\rangle| \geq M$ for all $\alpha \in$ $R^{+}$, then if we have a saturated chain of minimal length in strong Bruhat order from any $x=t^{\lambda} w \in \widetilde{W}$ to a pure translation, say $x \gg x_{1} \gg x_{2}>\cdots>x_{k}=t^{\mu}$, then $\nu_{x}=\mu^{+}$.

The key idea is to apply a convexity argument by extending Stembridge's definition of saturated sets in [Ste98], and then generalizing his Theorem 1.9 and Corollary 1.10 to include the non-integral case. To find a maximal pure translation element dominated by $x$, we employ the correspondence between certain edges in the quantum Bruhat graph and cocovers in Bruhat order. The following is a direct translation of Proposition 4.3 of Lam and Shimozono in [LS10].

Proposition 6.3. Let $\lambda \in Q^{+}$be dominant and superregular, and let $x=t^{v \lambda} w \in \widetilde{W}$. Then $y=$ $x t^{n v \alpha^{\vee}} r_{v \alpha} \lessdot x$ if and only if one of the following four conditions holds:

1. $\ell\left(v w_{0}\right)=\ell\left(v w_{0} r_{\alpha}\right)-1$ and $n=0$, in which case $y=t^{v r_{w_{0} \alpha}(\lambda)} r_{v w_{0} \alpha} w$.

2. $\ell\left(v w_{0}\right)=\ell\left(v w_{0} r_{\alpha}\right)+\left\langle\alpha^{\vee}, 2 \rho\right\rangle-1$ and $n=1$, in which case $y=t^{v r_{w_{0} \alpha}\left(\lambda+w_{0} \alpha^{\vee}\right)} r_{v w_{0} \alpha} w$.

3. $\ell\left(w^{-1} v w_{0}\right)=\ell\left(w^{-1} v w_{0} r_{\alpha}\right)+1$ and $n=\left\langle w_{0} \lambda, \alpha\right\rangle$, in which case $y=t^{v(\lambda)} r_{v w_{0} \alpha} w$.

4. $\ell\left(w^{-1} v w_{0}\right)=\ell\left(w^{-1} v w_{0} r_{\alpha}\right)-\left\langle\alpha^{\vee}, 2 \rho\right\rangle+1$ and $n=\left\langle w_{0} \lambda, \alpha\right\rangle+1$, in which case $y=$ $t^{v\left(\lambda+w_{0} \alpha^{v}\right)} r_{v w_{0} \alpha} w$.

Finally, repeated application of Proposition 6.3 then provides an interpretation for the maximal Newton polygon in terms of paths in the quantum Bruhat graph. 
Proposition 6.4. Let $\lambda \in Q^{+}$be dominant and superregular, and let $x=t^{v \lambda} w \in \widetilde{W}$. Then any saturated chain $x \gg x_{1} \gg x_{2} \gg \cdots \gg x_{k}=t^{\mu}$ of minimal length is canonically associated to a path of the same length in the quantum Bruhat graph from vwo to $w^{-1} v w_{0}$. Conversely, any path of minimal length, say length $k$, in the quantum Bruhat graph from vwo to $w^{-1} v w_{0}$ can be lifted to $2^{k}$ saturated chains in Bruhat order of minimal length from $x$ to a pure translation.

\section{Acknowledgements}

The author wishes to thank John Stembridge and Thomas Lam for helpful conversations in the early stages of this work. This project began while the author was partially supported by an NSF-sponsored Research Training Grant at the University of Michigan, and the author would like to thank the Combinatorics and Algebraic Geometry groups for their support.

\section{References}

[BDW96] Aaron Bertram, Georgios Daskalopoulos, and Richard Wentworth. Gromov invariants for holomorphic maps from Riemann surfaces to Grassmannians. J. Amer. Math. Soc., 9(2):529-571, 1996.

[Bea09] Elizabeth Beazley. Codimensions of Newton strata for $\mathrm{SL}_{3}(F)$ in the Iwahori case. Math. Z., 263(3):499-540, 2009.

[BFP99] Francesco Brenti, Sergey Fomin, and Alexander Postnikov. Mixed Bruhat operators and Yang-Baxter equations for Weyl groups. Internat. Math. Res. Notices, (8):419-441, 1999.

[BS12] Elizabeth Beazley and Luis Serrano. The poset of Newton polygons is shellable. Preprint in preparation, 2012.

[CdlOGP91] Philip Candelas, Xenia C. de la Ossa, Paul S. Green, and Linda Parkes. A pair of CalabiYau manifolds as an exactly soluble superconformal theory. Nuclear Phys. B, 359(1):21-74, 1991.

[Cha00] Ching-Li Chai. Newton polygons as lattice points. Amer. J. Math., 122(5):967-990, 2000.

[Dem72] M. Demazure. Lectures on $p$-divisible groups. In Lecture notes in mathematics, volume 302. Springer-Verlag, Berlin-New York, 1972.

[DL76] P. Deligne and G. Lusztig. Representations of reductive groups over finite fields. Ann. of Math. (2), 103(1):103-161, 1976.

[FW04] W. Fulton and C. Woodward. On the quantum product of Schubert classes. J. Algebraic Geom., 13(4):641-661, 2004.

[GHKR10] Ulrich Görtz, Thomas J. Haines, Robert E. Kottwitz, and Daniel C. Reuman. Affine Deligne-Lusztig varieties in affine flag varieties. Compos. Math., 146(5):1339-1382, 2010.

[Gro74] A. Grothendieck. Groupes de barsotti-tate et cristaux de Dieudonné. In Séminaire de Mathématiques Supérieures Été 1970, number 45, Montréal, Que., 1974. Les Presses de l'Université de Montréal. 
[Kat79] Nicholas M. Katz. Slope filtration of F-crystals. In Journées de Géométrie Algébrique de Rennes (Rennes, 1978), Vol. I, volume 63 of Astérisque, pages 113-163. Soc. Math. France, Paris, 1979.

[Kot85] Robert E. Kottwitz. Isocrystals with additional structure. Compositio Math., 56(2):201-220, 1985.

[Kot97] Robert E. Kottwitz. Isocrystals with additional structure. II. Compositio Math., 109(3):255339, 1997.

[LS10] Thomas Lam and Mark Shimozono. Quantum cohomology of $G / P$ and homology of affine Grassmannian. Acta Math., 204(1):49-90, 2010.

[Lus78] George Lusztig. Representations of finite Chevalley groups, volume 39 of CBMS Regional Conference Series in Mathematics. American Mathematical Society, Providence, R.I., 1978. Expository lectures from the CBMS Regional Conference held at Madison, Wis., August 8-12, 1977.

[Man63] J. I. Manin. Theory of commutative formal groups over fields of finite characteristic. Uspehi Mat. Nauk, 18(6 (114)):3-90, 1963.

[Maz72] B. Mazur. Frobenius and the Hodge filtration. Bull. Amer. Math. Soc., 78:653-667, 1972.

[Pet96] D. Peterson. Lectures on quantum cohomology of G/P. M.I.T., 1996.

[Pos01] Alexander Postnikov. Symmetries of Gromov-Witten invariants. In Advances in algebraic geometry motivated by physics (Lowell, MA, 2000), volume 276 of Contemp. Math., pages 251-258. Amer. Math. Soc., Providence, RI, 2001.

[Pos05] Alexander Postnikov. Quantum Bruhat graph and Schubert polynomials. Proc. Amer. Math. Soc., 133(3):699-709 (electronic), 2005.

[Rap05] Michael Rapoport. A guide to the reduction modulo $p$ of Shimura varieties. Astérisque, (298):271-318, 2005. Automorphic forms. I.

[RR96] M. Rapoport and M. Richartz. On the classification and specialization of $F$-isocrystals with additional structure. Compositio Math., 103(2):153-181, 1996.

[Ste98] John R. Stembridge. The partial order of dominant weights. Adv. Math., 136(2):340-364, 1998.

[Vie09] E. Viehmann. Truncations of level 1 of elements in the loop group of a reductive group. math.AG/0907.2331v1, 2009.

[Wit95] Edward Witten. The Verlinde algebra and the cohomology of the Grassmannian. In Geometry, topology, \& physics, Conf. Proc. Lecture Notes Geom. Topology, IV, pages 357-422. Int. Press, Cambridge, MA, 1995. 